\title{
Structural Optical Magnetic And Photocatalytic Properties of Ferromagnetic P-Type NiO: N-Type ZnO Nanocomposites
}

G. Kavitha

University of Madras

K. Thanigai Arul

AMET Deemed to be University

Manikandan Elayaperumal ( $\sim$ maniphysics@gmail.com )

Thiruvalluvar University https://orcid.org/0000-0002-3307-5776

\section{Research Article}

Keywords: Metal oxides, Nanoparticles, Magnetic, Photocatalytic activity.

Posted Date: December 6th, 2021

DOI: https://doi.org/10.21203/rs.3.rs-1117627/v1

License: (c) (i) This work is licensed under a Creative Commons Attribution 4.0 International License.

Read Full License 


\section{Abstract}

The semiconductor-transition conducting metal oxides (p-type NiO: n-type ZnO) nanocomposites (NCs) called (NZO) are successfully prepared by a simple wet-chemical route followed by the systematic sintering at different temperatures such as $400^{\circ} \mathrm{C}, 500^{\circ} \mathrm{C}, 600^{\circ} \mathrm{C}$, and $700^{\circ} \mathrm{C}$. The structure and morphology of the samples were characterized by X-ray diffraction (XRD), high-resolution scanning/transmission electron microscopy (HR-SEM/TEM), and energy-dispersive X-ray spectrometry (EDX) techniques. XRD analysis reveals that the average crystallite size of the NZO NCs was found to be in the range 16-18 $\mathrm{nm}$. The synthesized sample discloses a ferromagnetic behavior. The photocatalytic degradation of rhodamine B in an aqueous solution was superior at the NZO NC at $600{ }^{\circ} \mathrm{C}$ in comparison with other samples. Here, the NZO NCs display to be good candidates for magnetic and photocatalytic application.

\section{Introduction}

In the recent years, coupled metal oxide semiconductor nanomaterials have been received a lot of interest owing to its potential applications in optoelectronic devices, sensors, solar cells, photocatalysis and biological imaging, etc. [1-5]. It is well known that zinc oxide $(\mathrm{ZnO})$ is an efficient photocatalyst, with a band gap of around $3.2 \mathrm{eV}$ and it is one of the low-priced $n$-type semiconductors and a large exciton binding energy $(60 \mathrm{MeV})[6,7]$. Nickel oxide ( $\mathrm{NiO})$ is one of the most usually used photocatalysts with a wide range of applications as a p-type semiconductor with a wide band gap (3.6-4.0 eV) [8]. However, both the $\mathrm{ZnO}$ and $\mathrm{NiO}$ nanomaterials possess disadvantages of having a wide band gap $\leq 3.2 \mathrm{eV}$, therefore, the photo-excitation process requires UV region of the electromagnetic spectrum [9]. Nowadays, the coupled metal oxide semiconductors, i.e., two different metal oxides together act as a hetero-junction demonstrate improved photocatalytic activity. It is mainly due to the simultaneously excitation of the present metal oxide semiconductors, and improve their electron-hole pair separation, which enhances the photocatalytic efficiency [10]. Moreover, the efficiency of the photocatalytic activity depends upon the life time of the photo-generated electron/hole pairs [11]. A wide variety of synthesis routes have been used to prepare metal oxide nanoparticles, such as precipitation, microwave irradiation, mechanical milling, solution combustion, pulse laser deposition and vapour transparent deposition [12-14]. Different synthesis procedure yields different morphology of the sample such as nanorods, nanowire, nanoflower, nanocube and nanosheet, which altering its optical, electrical and magnetic properties have been reported [15-17].

Sun et al. [18] developed a novel dumb-bell shaped ZnO photocatalyst by hydrothermal method. The results indicated that the prepared dumbbell-shaped $\mathrm{ZnO}$ microcrystal exhibits superior photocatalytic activity and it could be considered as a promising photocatalyst for dye effluent degradation. Tong et al. [19] synthesized quasi-sphere-like ZnO by ice-water bath. This hierarchically nanostructured ZnO shows significantly improved photocatalytic activity than $\mathrm{ZnO}$ nanopowder. $\mathrm{ZnO}$ nanostructures in the shape of particles, rods, flower-like and micro-sphere synthesized using a facile hydrothermal method and the effect of morphology on the decolorization of acid red dye solutions under sunlight was studied by 
Mohajerania et al. [20]. Sobana et al. [21] reported that Ag NPs doped $\mathrm{TiO}_{2}$ for the degradation of direct red 23 and direct blue 53. Improved photocatalytic activity of $\mathrm{Ag}-\mathrm{TiO}_{2}$ was found to be due to electron trapping by Ag particle. Neppolian et al. [22] reported $\mathrm{ZrO}_{2}-\mathrm{TiO}_{2}$ binary oxide semiconductor nanoparticles for the application of photocatalyst.

In this present work, the $\mathrm{ZnO}-\mathrm{NiO} \mathrm{NCs}$ are prepared by wet chemical route and further the samples are sintered at different temperatures viz $400^{\circ} \mathrm{C}, 500^{\circ} \mathrm{C}, 600^{\circ} \mathrm{C}$ and $700^{\circ} \mathrm{C}$. The prepared samples were characterized by X-ray diffraction (XRD), high resolution scanning electron microscopy (HR-SEM), high resolution transmission electron microscopy (HR-TEM), diffuse reflectance spectroscopy (DRS) and vibrating sample magnetometer (VSM) and photocatalysis. Under light irradiation, sintered sample $\left(600^{\circ} \mathrm{C}\right)$ degrades rapidly due to higher transfer of electrons to conduction band from valence band and forming hole at valence band that providing superoxide for the degradation of the dye.

\section{Materials And Methods}

\subsection{Preparation}

Analytical reagent grade of nickel chloride, zinc acetate dihydrate and sodium hydroxide $(\mathrm{NaOH})$ were obtained from Merck and used without any purification. Nickel chloride $(1 \mathrm{M})$ and zinc acetate dihydrate $(1 \mathrm{M})$ and $\mathrm{NaOH}(2.5 \mathrm{M})$ were prepared using appropriate amount of distilled water separately in a beaker. Then, the solution of nickel chloride was kept on a magnetic stirrer and the zinc acetate solution $(1 \mathrm{M})$ was added drop wise to the nickel chloride solution. To the above mixture, the $\mathrm{NaOH}$ solution was added drop wise till the turbidity occurred for $3 \mathrm{~h}$. Thus, the green gel was formed that was washed thrice using distilled water. Eventually, the sample was permitted to dry in an oven for $3 \mathrm{~h}$ at $80^{\circ} \mathrm{C}$. In this step, the mixture of zinc hydroxide and nickel hydroxide was formed during the following reaction (1):

$\mathrm{Zn}\left(\mathrm{CH}_{3} \mathrm{COO}\right)_{2}(\mathrm{aq})+\mathrm{NiCl}_{2}(\mathrm{aq})+\mathrm{NaOH}(\mathrm{aq})$ à Ni(OH)$)_{2} . \mathrm{Zn}(\mathrm{OH})_{2}(\mathrm{~s})+\mathrm{CH}_{3} \mathrm{COONa}(\mathrm{aq})+\mathrm{NaCl}(\mathrm{aq})$

The ultimate precipitate was heated for $3 \mathrm{~h}$ in a muffle furnace at various temperatures namely, $400^{\circ} \mathrm{C}$, $500^{\circ} \mathrm{C}, 600^{\circ} \mathrm{C}$ and $700^{\circ} \mathrm{C}$ and denoted as NZO-400, NZO-500, NZO-600 and NZO-700 respectively. The following reaction (2) took place in the dehydration phase:

$\mathrm{Ni}(\mathrm{OH})_{2} \cdot \mathrm{Zn}(\mathrm{OH})_{2}(\mathrm{~s}) \diamond \mathrm{NiO} \cdot \mathrm{ZnO}(\mathrm{s})+\mathrm{H}_{2} \mathrm{O}(\mathrm{g})$

\subsection{Characterization}

The structural characterization of ZnO-NiO samples was performed using a Philips X'pert X-ray diffractometer with $\mathrm{CuK}_{\mathrm{a}}$ radiation at $\lambda=1.540 \AA$. Particle size and energy dispersive X-ray analysis of ZnO-NiO have been performed with a Jeol JSM6360 high resolution scanning electron microscopy. The transmission electron micrographs and photoluminescence of the samples were carried out by PhilipsTEM (CM20) and Varian Cary Eclipse Fluorescence Spectrophotometer respectively. The surface area 
was derived from the nitrogen adsorption-desorption isotherms using liquid nitrogen at $77 \mathrm{~K}$ using an automatic adsorption instrument (Quanta chrome Corp. Nova-1000 gas sorption analyzer). The magnetic properties of the samples were investigated using vibrating sample magnetometer (VSM) at room temperature in an applied magnetic field sweeping from -10000 to +10000 Oe using a PMC MicroMag 3900 model vibrating sample magnetometer (VSM) equipped with 1 Tesla magnet.

\subsection{Photocatalytic activity}

A photoreactor fitted with four $8 \mathrm{~W}$ medium pressure mercury vapor lamps emitting at $365 \mathrm{~nm}$ (Sankyo Denki, Japan) was used to determine the photocatalytic activities of the doped and undoped samples. The set of lamps was covered outside by a highly polished anodized aluminium reflector to preserve the light intensity. A borosilicate glass tube of $15 \mathrm{~mm}$ inner diameter placed at the centre of the photoreactor was used as the reaction vessel. The reactor was cooled by fans that mounted inside it. The light intensity was determined by ferrioxalate actinometry. Fresh solution of rhodamine B was used for the photodegradation. After the addition of the nanoparticles to the dye solution, air was bubbled through the solution and, further the nanoparticles are stirred at constant motion. The dissolved oxygen was measured by an Elico dissolved oxygen analyzer PE 135. After the illumination, the nanoparticles were recovered by centrifugation and the dye was analyzed by spectrophotometrically at $552 \mathrm{~nm}$. The calibration curve was constructed by measuring the absorbance of rhodamine $B$ at different ppm.

\section{Results And Discussion}

\subsection{X-ray diffraction}

The crystal structure and phase purity of $\mathrm{ZnO}-\mathrm{NiO}$ nanoparticles are analyzed by $\mathrm{X}$-ray diffraction. The XRD patterns of the as-synthesized ZnO-NiO nanoparticles are shown in Fig. 1. The diffraction peaks are corresponding to both ZnO (JCPDS No. 36-1451) [23] and NiO (JCPDS No. 47-1049) [24] are noticed. The peaks around $37^{\circ}, 43^{\circ}, 62.4^{\circ}$ and $74.7^{\circ}$ are corresponding to the NiO planes (111), (200), (220) and (311). Similarly, the peaks around $31.6^{\circ}, 34.2^{\circ}, 36.19^{\circ}, 47.4^{\circ}, 56.6^{\circ}, 67^{\circ}, 68.9^{\circ}$ and $78.8^{\circ}$ are related to the planes of ZnO (100), (002), (101), (102), (110), (112), (201) and (202) respectively. As the temperature increases, the intensity of diffraction peaks is enhanced that revealing high crystallinity. The peak around $36.19^{\circ}$ is split and its intensity varied with the sintering temperature due to the modulation of crystal growth orientation. Here, the planes of $\mathrm{NiO}$ are predominant compared with the planes of $\mathrm{ZnO}$. Similar trend was explained in the composite of $\mathrm{ZrO}_{\mathrm{x}} / \mathrm{ZnO}$ metal oxides by hydrothermal method [24].

The average crystallite size was calculated using Scherrer's formula given in Eq. (1)

$$
L=\frac{0.89 \lambda}{\beta \cos \theta}
$$

where $L$ is the crystallite size, $\lambda$, the $X$-ray wavelength, $\theta$, the Bragg diffraction angle and $\beta$, the full width at half maximum (FWHM). From the Table-1 it was clear that as the temperature increases the average 
crystallite size gradually increases from 16 to $26 \mathrm{~nm}$. XRD pattern of pure and nanocomposites of NZO diffraction were given in the supplementary materials.

\section{Table -1: Particles Size Determination of HTCMOs NCs}

\begin{tabular}{|c|c|c|}
\hline $\begin{array}{c}\text { Calcination } \\
\text { Temperature }\left({ }^{\circ} \mathrm{C}\right)\end{array}$ & \multicolumn{2}{|c|}{$\begin{array}{c}\text { Particle Size (nm) } \\
\text { [Characteristic Peaks] }\end{array}$} \\
\cline { 2 - 3 } & Zn0 & NiO \\
\hline 300 & 10.087 & 10.873 \\
\hline 400 & 16.135 & 16.135 \\
\hline 500 & 17.611 & 18.261 \\
\hline 600 & 20.175 & 20.174 \\
\hline 700 & 25.525 & 26.913 \\
\hline
\end{tabular}

\subsection{Morphological analysis}

The morphology of the prepared ZnO-NiO was examined by SEM. Growth of the nanoparticles cannot be controlled in this method leading to the variation of particle sizes. Fig. 2a shows the SEM micrographs of $\mathrm{ZnO}-\mathrm{NiO}$ nanoparticles, which are of irregular spherical shape with varying sizes between 15 and $20 \mathrm{~nm}$. The composition of ZnO-NiO nanoparticles was analyzed by energy dispersive X-ray analysis (EDX). The EDX results showed the presence of $\mathrm{Zn}, \mathrm{Ni}$ and $\mathrm{O}$ without any other characteristic peaks (Fig. 2b). The HRTEM image showed the presence of abundant nearly flakes-like particles that were self-assembled (Fig. 2c) and the lattice fringes of $\mathrm{ZnO}-\mathrm{NiO}$ nanoparticle are shown in Fig. 2d. The flakes-like particles have an average size in the range $16-18 \mathrm{~nm}$.

\subsection{VSM}

Magnetization measurements on the synthesized nanoparticles were carried out and shown in Fig. 3. A weak ferromagnetic behaviour of the synthesized sample (NZO-400) was confirmed by the shape of hysteresis loop. From the VSM measurements, the coercive field $\left(\mathrm{H}_{\mathrm{c}}\right)$ and the remanent magnetization $\left(M_{r}\right)$ of the sample were estimated to be $27.47 \mathrm{O}_{\mathrm{e}}$ and $0.004 \mathrm{emu} / \mathrm{g}$ respectively. The low $\mathrm{H}_{\mathrm{c}}$ and $\mathrm{M}_{\mathrm{r}}$ confirmed the soft and weak ferromagnetic nature of the sample. The saturation magnetization $\left(\mathrm{M}_{\mathrm{s}}\right)$ of 
sample was found to be $0.200 \mathrm{emu} / \mathrm{g}$ which confirmed its ferromagnetic behaviour. Because of sintering, the particle size was reduced thereby formation of different magnetic domain which orient in the direction of magnetic field. The ferromagnetic behavior of the nanocomposite can be explained by dimensional factor as well as generation of $\mathrm{Zn}_{1-x} \mathrm{Ni}_{x} \mathrm{O}$ solid solution. The present results coincide with the results obtained by Schwartz et al $[25,29]$.

\subsection{Photocatalytic activity}

In order to determine the photocatalytic activity of coupled metal oxides ZnO-NiO NCs, a series of experiments were carried out with rhodamine $B$ in aqueous suspension under the light of wavelength 552 $\mathrm{nm}$. The photocatalytic degradation follows a pseudo-first order reaction and its kinetics can be expressed using $\ln \left(C_{0} / C_{t}\right)=k t$, where $k$ is the apparent reaction rate constant, $C_{0}$ is the initial concentration of rhodamine $B$, the reaction time $(t)$ and $C_{t}$ is the concentration of rhodamine $B$ at time t. Fig. 4 shows the kinetic fit for the degradation of rhodamine B over the coupled ZnO-NiO NCs with the catalyst dosage of $30 \mathrm{mg}$, rhodamine $\mathrm{B}$ concentration of $75 \mathrm{mg} / \mathrm{L}$ and at $\mathrm{pH} 5$. At $600^{\circ} \mathrm{C}$, the sample rapidly degrades the dye owing to higher transfer of electrons to conduction band from valence band and forming hole at valence band under light irradiation that providing superoxide to the dye compared with other samples [26]. Here, the $\mathrm{hVB}^{+}$(hole at valence band) and $\mathrm{eCB}^{-}$(electron at conduction band) are dominant

oxidizing and reducing agents, respectively. The $\mathrm{hVB}^{+}$interacts with the dye (organic compounds) leading to form $\mathrm{CO}_{2}$ and $\mathrm{H}_{2} \mathrm{O}$ as end products. The $\mathrm{hVB}^{+}$also oxidizes the organic compounds by interacting with water for the generation of hydroxyl radical $(\cdot \mathrm{OH})$. This radical is an electrophilic nature that can oxidize all electron rich organic molecules to convert them to $\mathrm{CO}_{2}$ and water. The photocatalytic degradation of organic pollutants is a crucial one that can be achieved effectively by the process of reduction of oxygen and the oxidation of the dye that are continued simultaneously in order to avoid the build up of electron in the conduction band. This leads for the reduction of the rate of recombination of $\mathrm{eCB}^{-}$and $\mathrm{hVB}^{+}$[27-28] and the reaction rate given in the supplementary materials.

\section{Conclusions}

In the present work, the ZnO-NiO NCs are successfully prepared by a simple wet chemical route with cost effective manner in the RT. It was found that the generation of NZO NCs commences at $400^{\circ} \mathrm{C}$ and as the temperature rises, the size of the composites gradually reduced. The flakes like morphology were observer from the electron microscope from the synthesized NZO NCs were confirmed by HR-SEM and HR-TEM analyses. The magnetization versus field strength behaviors of curve of ZnO-NiO NCs showed a hysteresis characteristic of ferromagnetic materials due to the dimensional effect of $\mathrm{NiO}$ nanoparticles. Besides, the ZnO-NiO NCs showed high photocatalytic efficiency for the degradation of rhodamine $B$ dye under the light irradiation. Thus, the prepared $\mathrm{ZnO}-\mathrm{NiO}$ sample is a potential candidate for photocatalytic applications.

\section{Declarations}


Acknowledgement:

Author (E.M.) grateful to the UNESCO-UNISA AFNET, University of South Africa (UNISA), Pretoria, South Africa for providing facilities to carry out this research work.

\section{Informed Consent}

Informed consent was obtained from all individual author for this study.

\section{Authors' Contributions}

GK aimed and conceived of the study, formulated its design, coordinated the conduct of the study including materials synthesis, data collection, analysis and interpreted the data, and drafted the manuscript; KT participated in the design of the study, assisted in interpreting the data, and helped to draft the manuscript; EM and JK participated in the design of the studies like magnetic and electron microscopic measurement etc assisted in interpreting the data, and helped to draft the manuscript. All authors read and approved the final manuscript.

\section{Compliance with Ethical Standards}

\section{Conflicts of Interest}

The authors report no conflicts of interest. When the editor or individual reader's need the original scientific data/images needed from this study. We will provide based on the genuine and ethical standard.

\section{Research Data Policy and Data Availability Statements}

The data that support the findings of this study are available from, but restrictions apply to the availability of these data, which were used under license for the current study, and so are not publicly available. Data are however available from the authors upon reasonable request and with permission.

\section{References}

[1] A. P. Subramanian, S. K. Jaganathan, A. Manikandan, K. N. Pandiaraj, N. Gomathi, E. Supriyanto, RSC Adv. 6, (2016) 48294-48314.

[2] M. V. Vellayappan, S. K. Jaganathan, A. Manikandan, RSC Adv. 6, (2016) 114859-114878.

[3] V. Muthuvignesh, V. J. Reddy, S. Ramakrishna, S. Ray, A. Ismail, M. Mandal,

A. Manikandan, S. Seal and S. K. Jaganathan, RSC Adv. 6 (2016) 83638 -83655.

[4] G Bupesh, E Manikandan, K Thanigaiarul, S Magesh, V Senthilkumar, S Tamilarasan, K Pandian, A Gurib-Fakim, M Maaza, J. Nanomed. Nanotechnol. 7 (355) 2 (2016). 
[5] AH Shah, E Manikandan, MB Ahmed, V Ganesan, J. Nanomed. Nanotech. 43 (2013).

[6] A. Manikandan, J. Judith Vijaya, S. Narayanan, L. John Kennedy, J. Nanosci. Nanotech. 14 (2014) 2507-2514.

[7] A. Manikandan, J. Judith Vijaya, C. Ragupathi, L. John Kennedy, J. Nanosci. Nanotech. 14 (2014) 2584-2590.

[8] A. Manikandan, L. John Kennedy, J. Judith Vijaya, Physica E, 49 (2013) 117-123.

[9] M.R. Hoffmann, S. T. Martin, D. W. Bahnemann, Chem. Rev. 95 (1995) 69-96.

[10] C. Karunakaran, R. Dhanalakshmi, P. Gomathisankar, G. Manikandan, J. Hazard. Mater. 176 (2010) 799-86.

[11] H. H. Mohamed, D. W. Bahnemann, Appl. Catal. B: Environ. 128 (2012) 91-104.

[12] Z. Wang, X.F. Qian, J. Yin, Z.K. Zhu, Langmuir 20 (2004) 3441.

[13] P.M. Aneesh, K.A. Vanaja, M.K. Jayaraj, Nanopht. Mater. IV 6639 (2007) 66390.

[14] A.P.A. Oliveira, J.F. Hochepied, F. Grillon, M.H. Berger, Chem. Mater. 15 (2003) 3202.

[15] Z.Q. Li, Y.J. Xiong, Y. Xie, Inorg. Chem. 42 (2003) 8105.

[16] X. Wang, Y. Ding, Z. Li, J. Song, Z.L. Wang, J. Phys. Chem C. 113 (2009) 1791.

[17] D. Wang, C. Song, J. Phys. Chem. B. 109 (2005) 12697.

[18] J. H. Sun, S. Y. Dong, Y. K. Wang, S. P. Sun, J. Hazard. Mater. 172 (2009) 1520.

[19] Y. Tong, J. Cheng, Y. Liub, G. G. Siu, Scr. Mater. 60 (2009) 1093.

[20] M. S. Mohajerania, A. Laka, A. Simchia, J. Alloys Compd. 485 (2009) 616.

[21] N. Sobana, M. Muruganadham, M. Swaminathan J. Mol. Catal. A: Chem. 258 (2006) 124.

[22] B. Neppolian, Q. Wang, H. Yamashita, H. Choi, Appl. Catal. A 333 (2007) 264-271.

[23] P. Basnet, D. Samanta, T, Inakhunbi Chanu, et al. SN Appl. Sci. 1 (2019) 633.

[24] H. Yan, D. Zhang, J. Xu, et al. Nanoscale Res Lett 9, (2014).424.

[25] W. Liao, T. Zheng, P. Wang, S. Tu, W. Pan, Efficient microwave-assisted photocatalytic degradation of endocrine disruptor dimethyl phthalate over composite catalyst ZrOx/ZnO, J. Environ. Sci. 22 (2010) 1800-1806. 
[26] D. A. Schwartz, N. S. Norberg, Q. P. Nguyen, J.M.Parker, D.R. Gamelin, J. Am. Chem. Soc. 125 (2003) 13205-13218.

[27] M. Boroski, A.C. Rodrigues, J.C. Garcia, L.S. Sampaio, J. Nozaki, N. Hioka, Journal of Hazardous Materials 162 (2009) 448-454.

[28] M.R. Hoffmann, S.T. Martin W. Choi, D.W. Bahnemann, Chemical Reviews 95 (1995) 69-96.

[29]. G. Kavitha, K.T. Arul, P. Babu, Enhanced acetone gas sensing behaviour of $n-\mathrm{ZnO} / p-\mathrm{NiO}$ nanostructures. Journal of Materials Science: Materials in Electronics, 2018, 29(8), pp. 6666-6671.

\section{Figures}
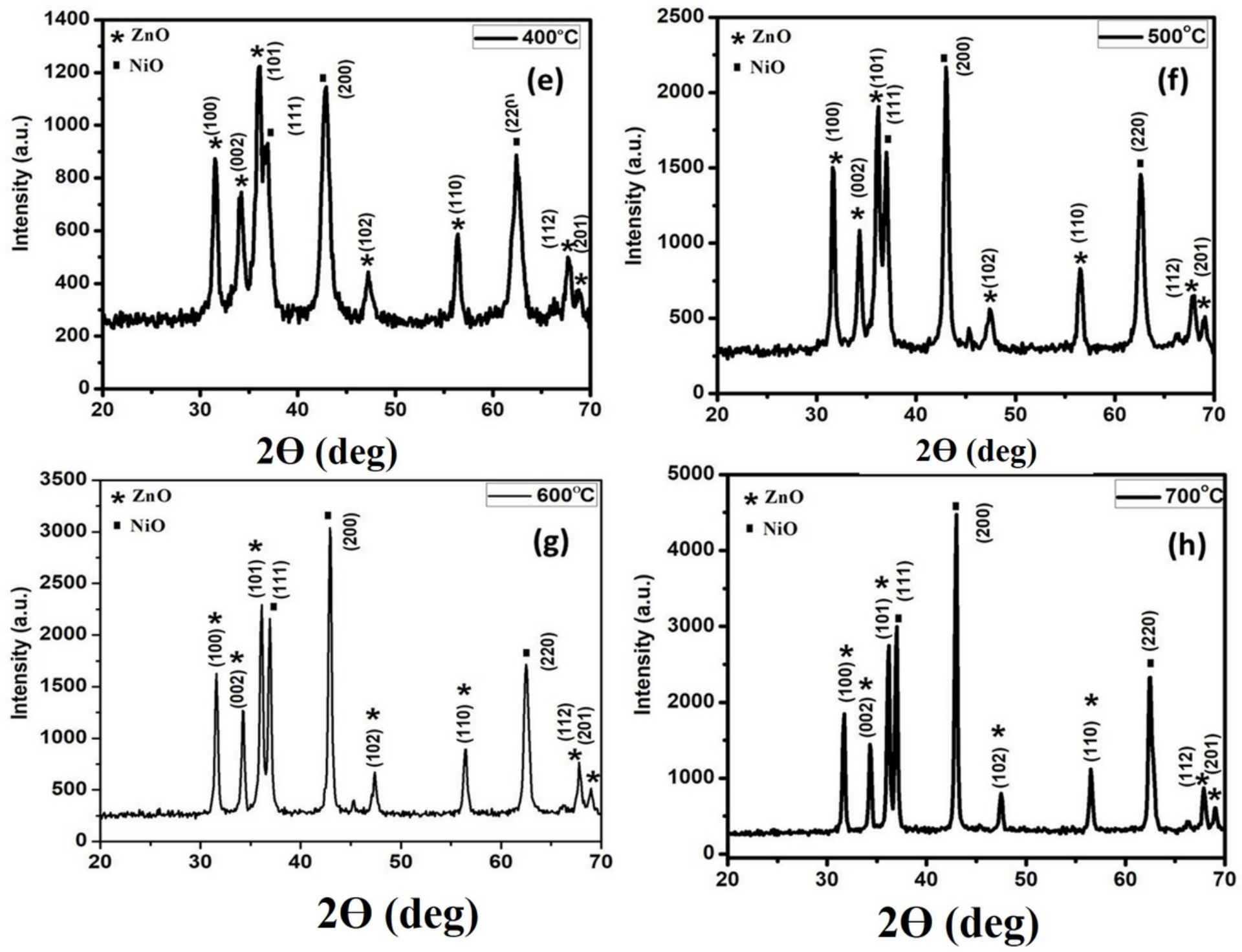

Figure 1

XRD patterns of ZnO-NiO nanocomposites of (e) NZO-400, (f) NZO-500, (g) NZO-600 and (h) NZO-700 

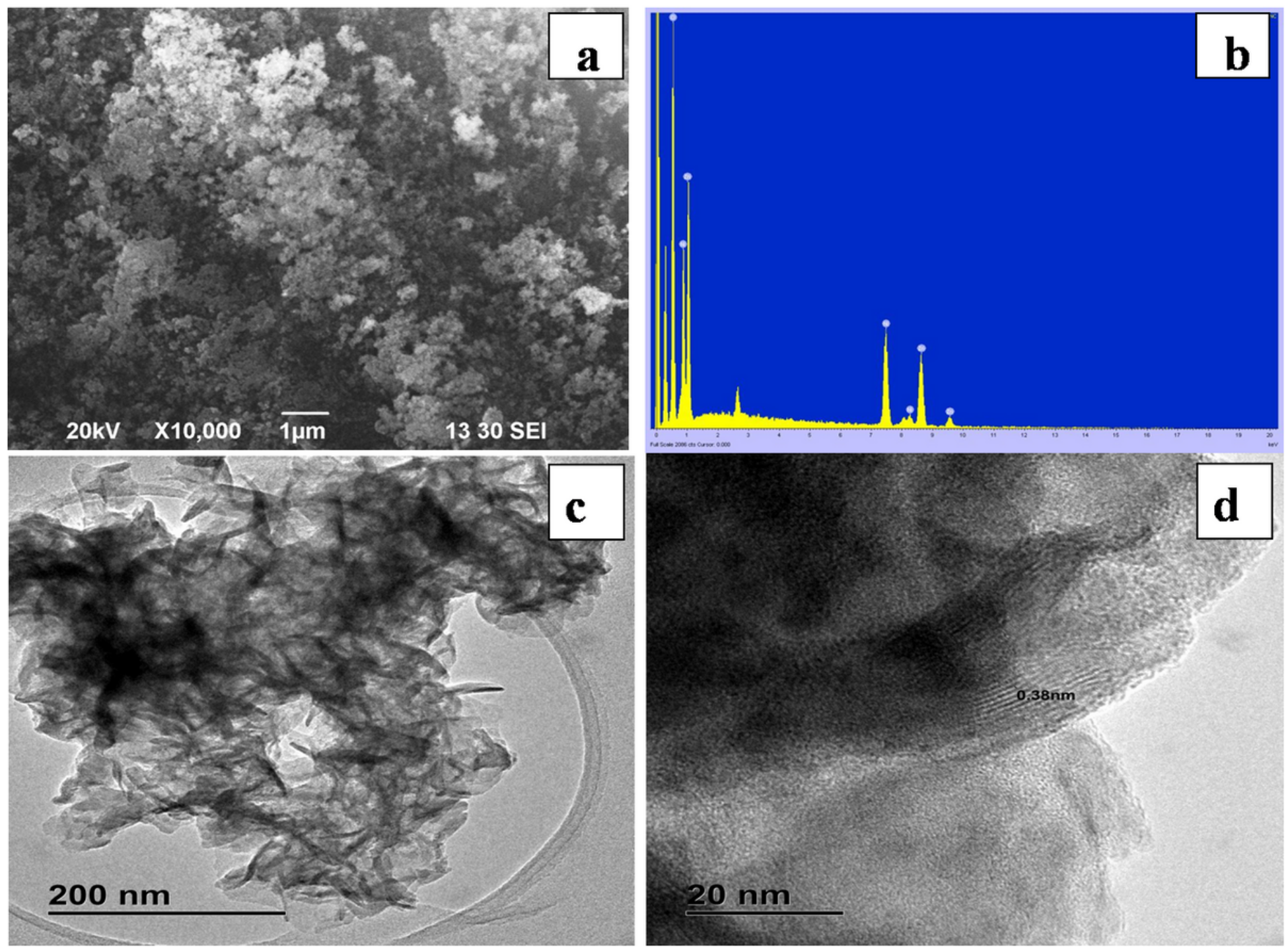

Figure 2

ZnO-NiO nanocomposite (a) SEM image (b) EDX, (c) HR-TEM image and (d) lattice fringes. 


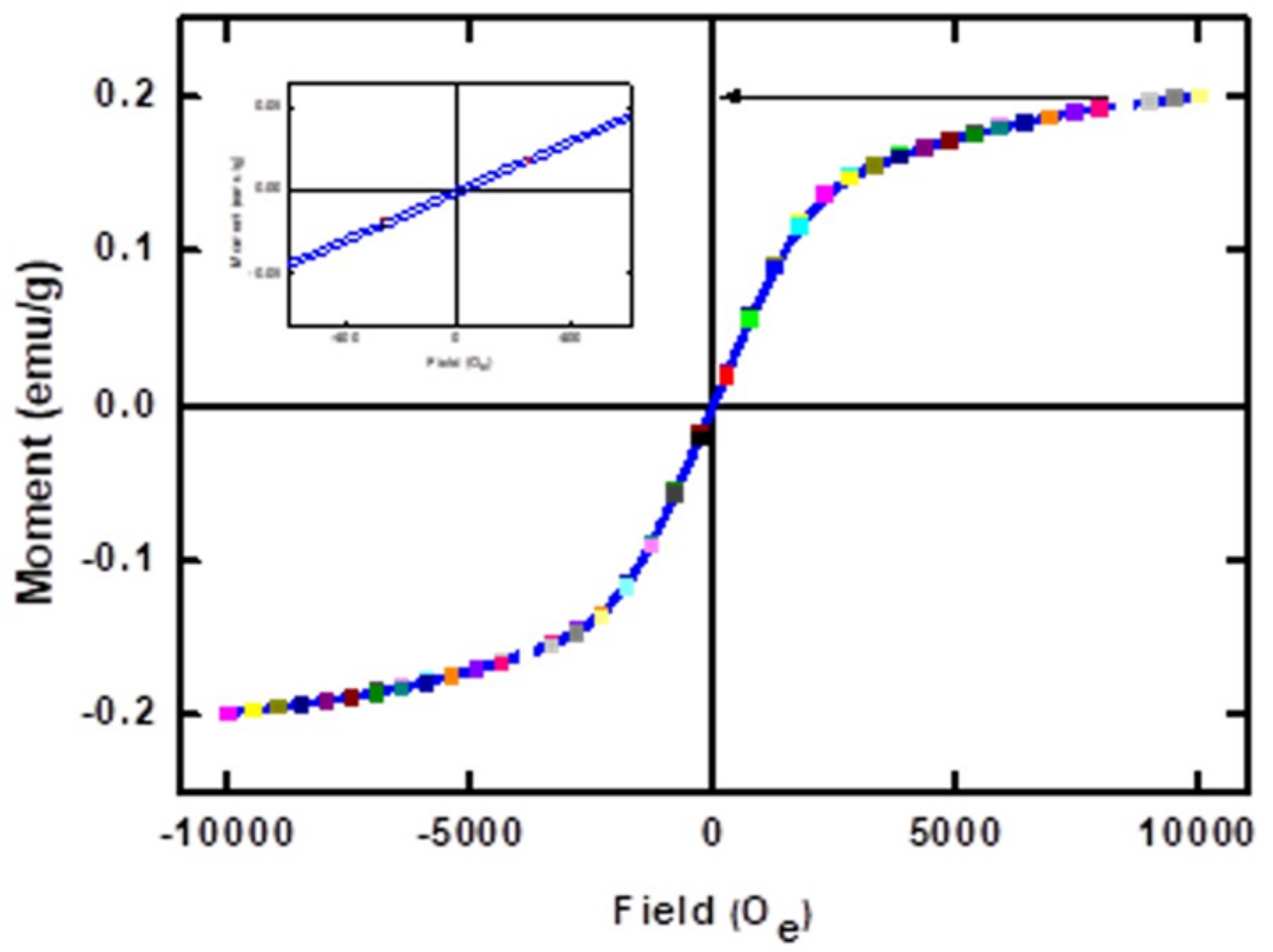

Figure 3

Magnetic hysteresis loop of $\mathrm{ZnO}-\mathrm{NiO}$ nanocomposites at $400{ }^{\circ} \mathrm{C}$. 


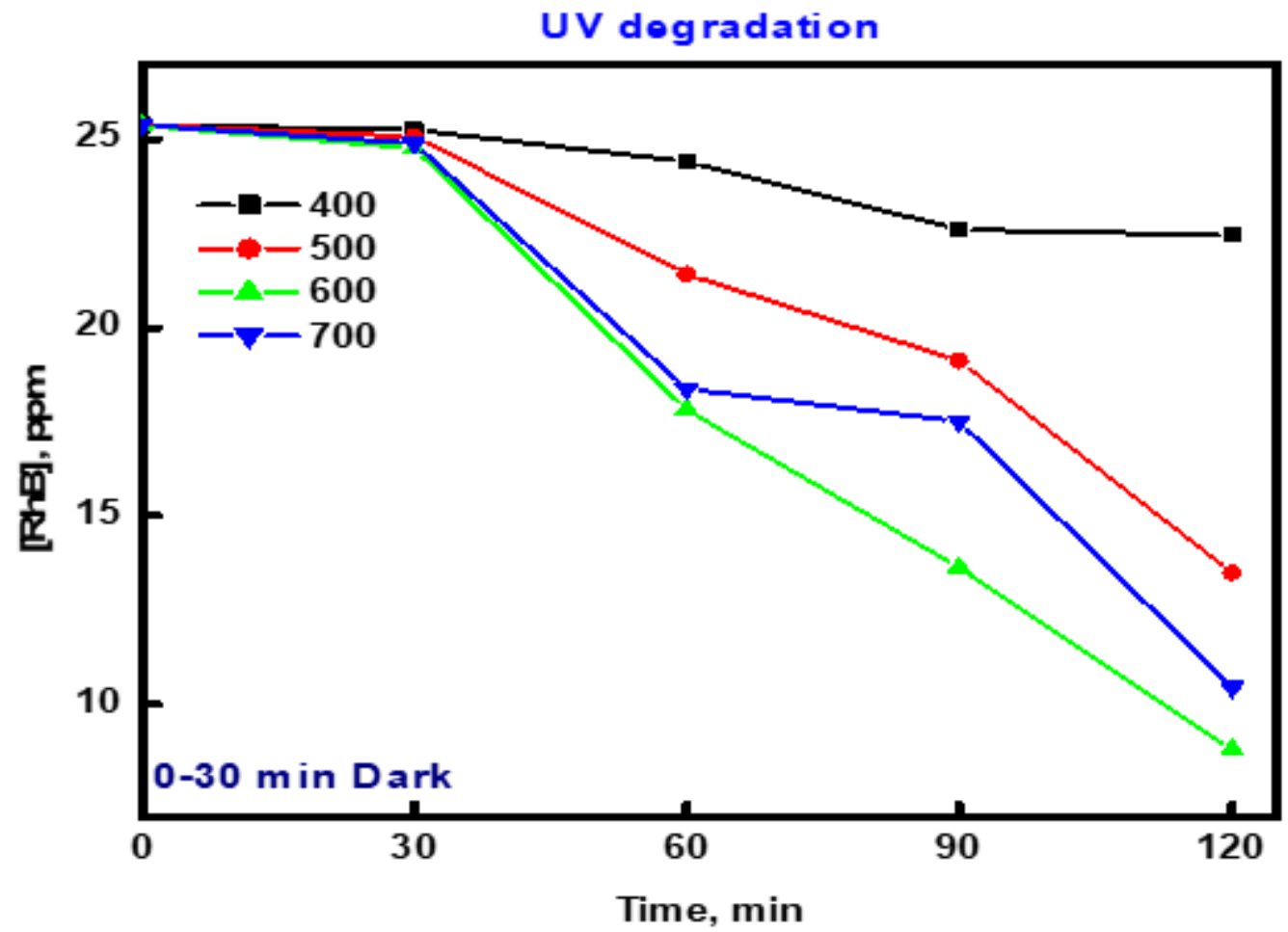

Figure 4

PCD efficiency (\%) of ZnO-NiO nanocomposites.

\section{Supplementary Files}

This is a list of supplementary files associated with this preprint. Click to download.

- GraphicalAbst.jpg

- Supplementararymaterials.doc 